\title{
Determining the speed of soil particles during the covering of set onion with a disk-type working element featuring a soil guide
}

\author{
Alexey Dorokhov ${ }^{1}$, Alexander Aksenov ${ }^{1}$, Maksim Mosyakov ${ }^{1 *}$, and Nikolay Sazonov ${ }^{1}$ \\ ${ }^{1}$ Federal Scientific Agroengineering Center VIM, 5, 1st Institutskiy proezd, 109428, Moscow, Russia
}

\begin{abstract}
In Russia, the major share of vegetable products is produced during the period of March to August. This is due to the inadequate volumes of vegetables fit for long-term storage, and the use of technologies of early production of vegetable cultures. Apart from the above, vegetable farming is highly dependent on imported seed stock. In view of the above, import phaseout and improvement of competitiveness of vegetable cultures in Russia should focus on the development of technologies of production of storable products, as well as on methods of early harvesting of vegetables (during the period of May to July). With regards to the production of bulb vegetables, this problem may be solved by expanding industrial cultivation of onions from set onions, as well as planting of both seeds and seedlings during the autumn season. In particular, techniques of production of set onion, bulb onion from seedlings, and of blackseed onion have poor scientific and methodological background. This deficiency results in a gap between domestic and international process and engineering aspects. This calls for the development and introduction of machines designed for production of onion from seedlings that would be in line with the modern level of development of farm machinery industry for vegetable production.
\end{abstract}

\section{Introduction}

Results of studies of various techniques of bulb culture planting witness that maximum yield may be achieved by way of planting bulbs and their subsequent covering with their stem plate down.

Modern level of development of means of mechanizations of bulb culture planting allows to state that planter machinery manufacturers have solved the problem of translating bulbs from random to the required oriented position during planting, using certan improvements of the planting mechanism [1-4].

However, according to the process scheme of sowing or planting machines involves, at the final stage of planting, the operation of bulb covering. Quality performance of this operation has a critical effect on the engineering complex of interrelated operations of bulb planting. Consequently, an imperfect design of covering working elements of an onion

* Corresponding author: Maks.Mosyakov@yandex.ru 
planter impairs the planter's quality characteristics of operation and thus does not ensure full compliance with agrotechnical requirements during bulb planting [5-8].

The main drawback of covering implements of sowing and planting machines is their action on the planting stock in the longitudinal direction which results in piling up of the surface layer of soil and the planting stock itself in the direction of the planter's movement. This, in turn, reduces the uniformity of distribution of bulbs within a furrow and the effectiveness of orientation of bulbs with their stem plate downward by the planter [9-12].

In order to eliminate these drawbacks, the team of FGBNU «Federal Scientific Agroengineering Center VIM» developed a disk-type covering working element featuring soil guides which acts on the sowing seed stock vertically, i.e., soil is directed onto bulbs vertically, unlike some known designs of covering working elements that act on the bulb longitudinally due to shifting of a layer of soil along the direction of bulb planting.

The disk covering working element (Figure 1) is comprised of a concave disk 1 and soil guides 2 .
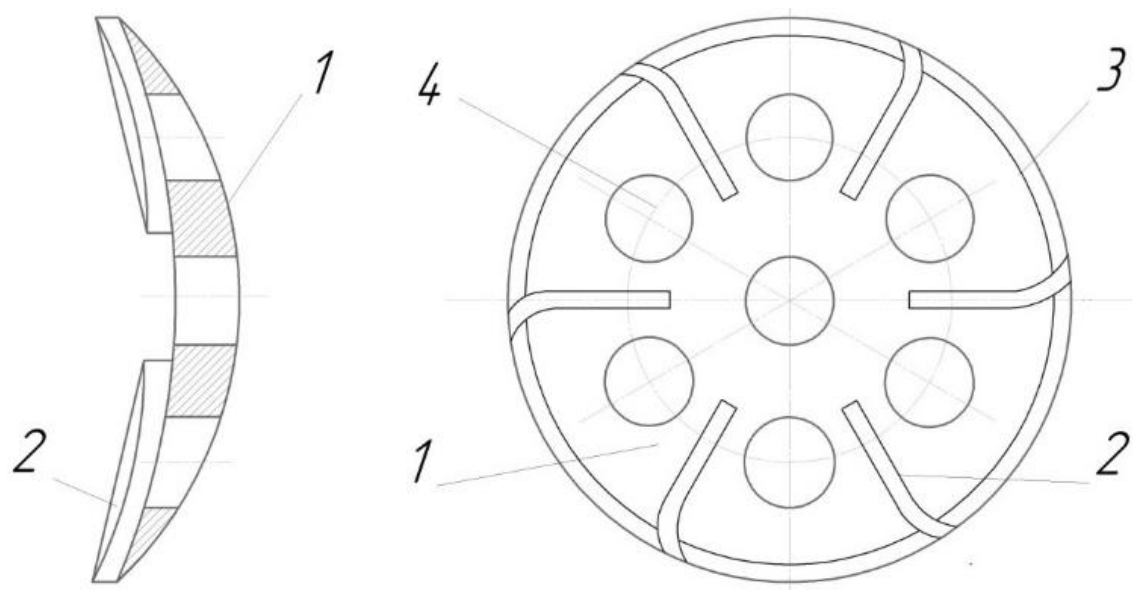

Fig. 1. Design of disk covering working element with soil guides: 1 - concave disk; 2 - soil guides; 3 - cutting edge; 4 - round apertures

The disk has cutting edge 3 and round apertures 4 located at its work surface that additionally facilitate soil loosening and prevent soil from sticking to the disk.

Covering of bulbs during their planting into the furrow by the covering element with soil guides is performed as the result of interaction of the disk element with the soil, which acts on the bulbs only from transversal and vertical directions, and prevents any action on bulbs in the longitudinal direction thus ensuring its initial position is preserved.

Due to the altered nature of interaction of the soil guides with the soil, bulbs are covered effectively, and the soil is additionally loosened.

\section{Methods and methods}

To study movement of soil particle B after leaving the surface of the soil guide, we shall use the theory of flight of a body thrown at angle $\gamma$ to the horizon (Figure 2). Disregarding air resistance, let us write the equation of movement of soil particle $B$ as follows [13-15]:

$$
\left\{\begin{array}{l}
x=v_{B o} \cos \gamma t, \\
z=v_{B o} \sin \gamma t-\frac{t^{2}}{2} .
\end{array}\right.
$$


where $\mathrm{v}_{\mathrm{Bo}}-$ is the initial flight speed of soil particle $\mathrm{B}$ at a specific moment in time $\mathrm{t}=$ $0, \mathrm{~m} / \mathrm{s} ; \gamma-$ and $\mathrm{v}_{\mathrm{Bo}}$ is the angle of inclination of speed to the horizon, degrees.

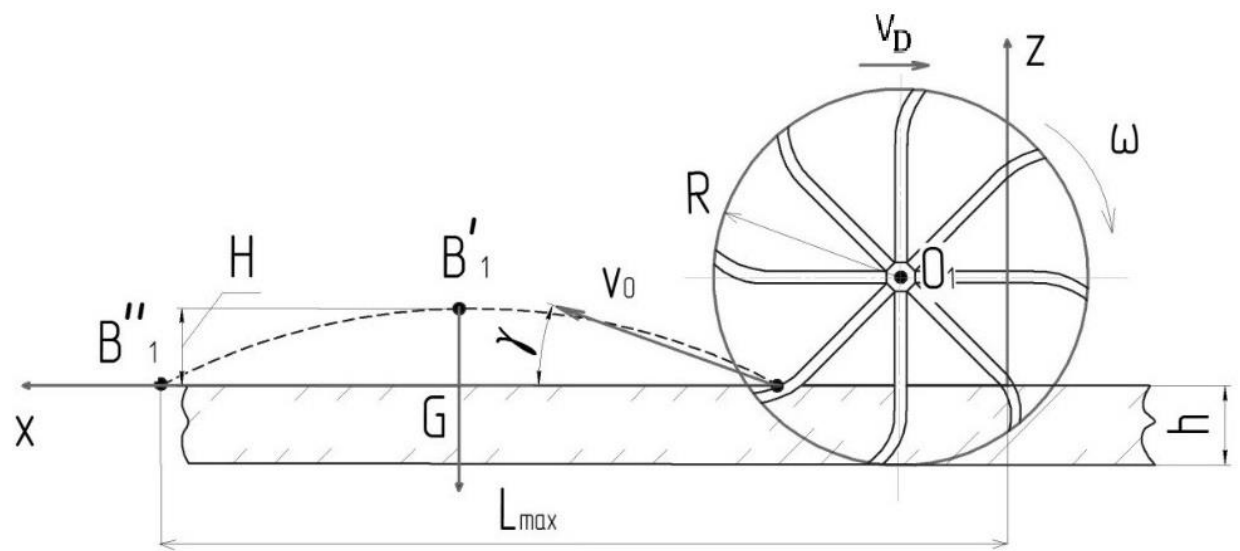

Fig. 2. Determining flight distance of a soil particle.

Initial flight speed $\mathrm{v}_{\mathrm{Bo}}$ of soil particle $\mathrm{B}$ at a moment in time is determined $\mathrm{t}=0$ using the expression:

$$
\mathrm{v}_{\mathrm{Bo}}=\sqrt{\dot{\mathrm{x}}_{1}^{2}+\dot{\mathrm{y}}_{1}^{2}+\dot{\mathrm{z}}_{1}^{2}+\mathrm{v}_{\mathrm{D}}^{2}+2 \mathrm{v}_{\mathrm{D}} \dot{\mathrm{x}}_{1}},
$$

where $v_{D}-$ is travel speed of the disk covering device, $\mathrm{m} / \mathrm{s}$.

Since according to Figure 1 annular velocity $\mathrm{v}_{\mathrm{B} e}$ of particle $\mathrm{B}$ can be expressed as:

$$
\mathrm{v}_{\mathrm{Be}}=\mathrm{v}_{\mathrm{D}} \cos \alpha,
$$

the travel speed $v_{D}$ of disk covering element can be expressed as follows:

$$
\mathrm{v}_{\mathrm{D}}=\frac{\mathrm{v}_{\mathrm{Be}}}{\cos \alpha}=\frac{\pi \mathrm{nR}}{30 \cos \alpha^{\prime}}
$$

where $\mathrm{n}-$ is the rate of rotation of the disk covering element's shaft, $\mathrm{min}^{-1}$; $\mathrm{R}-$ is the radius of the disk covering element, $m$.

Taking into consideration the expression (4), formula (2) can be expressed as follows:

$$
\mathrm{v}_{\mathrm{Bo}}=\sqrt{\dot{\mathrm{x}}_{1}^{2}+\dot{\mathrm{y}}_{1}^{2}+\dot{\mathrm{z}}_{1}^{2}+\left(\frac{\pi \mathrm{nR}}{30 \cos \alpha}\right)^{2}+2\left(\frac{\pi \mathrm{nR}}{30 \cos \alpha}\right) \dot{\mathrm{x}}_{1}}
$$

To determine the initial flight speed of soil particle $v_{0}(2)$, the rate of rotation of the disk covering element $\mathrm{n}$ was determined. This value is used in the evaluation formula (5).

The rate of rotation of the disk covering element was determined using a laboratory setup (Figure 3) at various values of travel speed of the disk covering element $v_{d}$ and varying values of angle of attack $\alpha$; the center-to-center distance $S$ between disks of the disk covering element remained unchanged and was equal to $S=0.12 \mathrm{~m}$. 


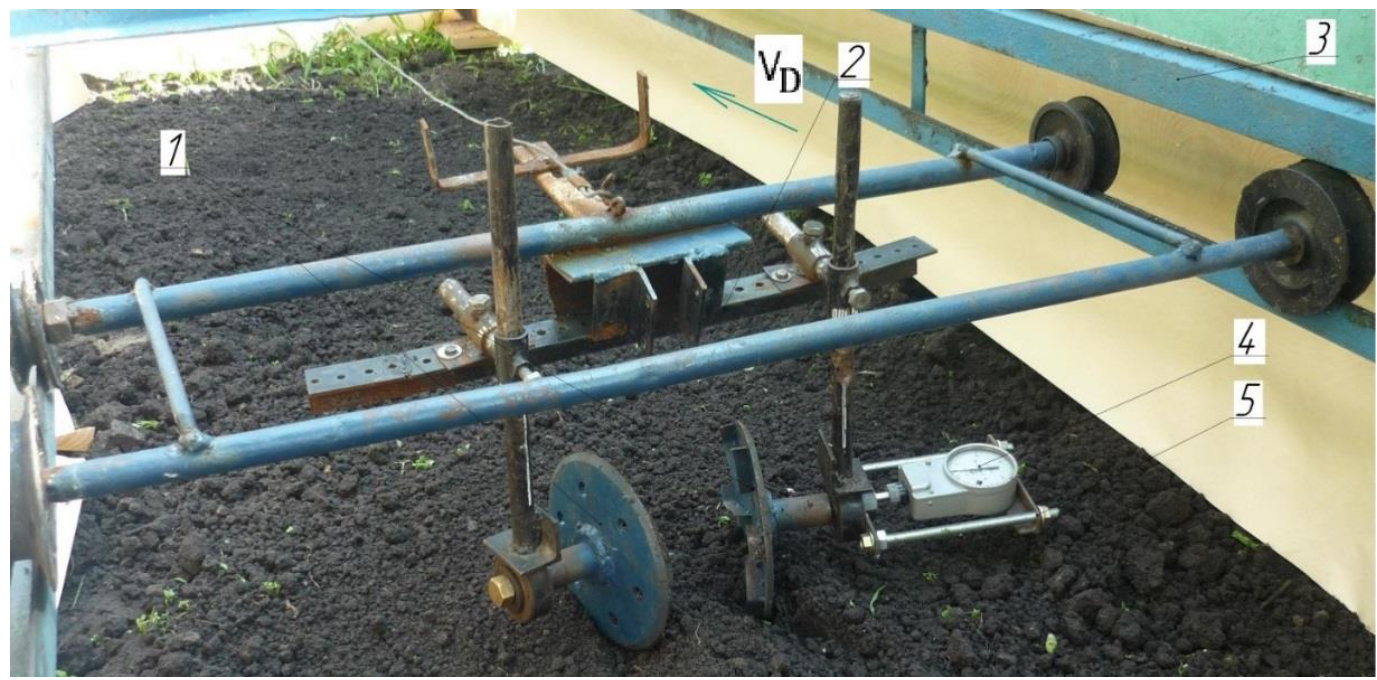

Figure 3. Diagram of laboratory setup for determining the rate of rotation of the disk covering element: 1 - disk covering element; 2 - driving trolley; 3 - soil channel; 4 - tachymeter; 5 mounting prop.

The lower boundary of the travel speed variation interval $\mathrm{v}_{\mathrm{D}}$ had been selected to be equal to $0.8 \mathrm{~m} / \mathrm{s}$, and subsequently increased at the increment of $0.2 \mathrm{~m} / \mathrm{s}$, up to the threshold value of $1.2 \mathrm{~m} / \mathrm{s}$.

The angle of attack of disks $\alpha$ was varied from 5 to $30^{\circ}$ with the variation interval of 5 degrees.

The research procedure was as follows (Figure 4).

Disk covering device 1 was installed on driven trolley 2 of mobile soil channel 3 . Tachymeter was securely fixed at the shaft of the disk covering element at mounting prop 5. Following that, trolley 2 was set in motion, and values of rate of rotation of the shaft of the disk covering element, with values previously set to $v_{D}$ and $\alpha$.

The measurement range of the rate of rotation of the disk covering element 1 provided by tachymeter 4 was set within the range of 10 to $300 \mathrm{~min}^{-1}$, by rotating adjustment screw 2. The degree of frictional interaction of shaft 3 of the disk covering element 1 with the wetted tip 6 of tachymeter 4 was adjusted by moving stand 5 along guides 7 and securing it with threaded joint 8 .

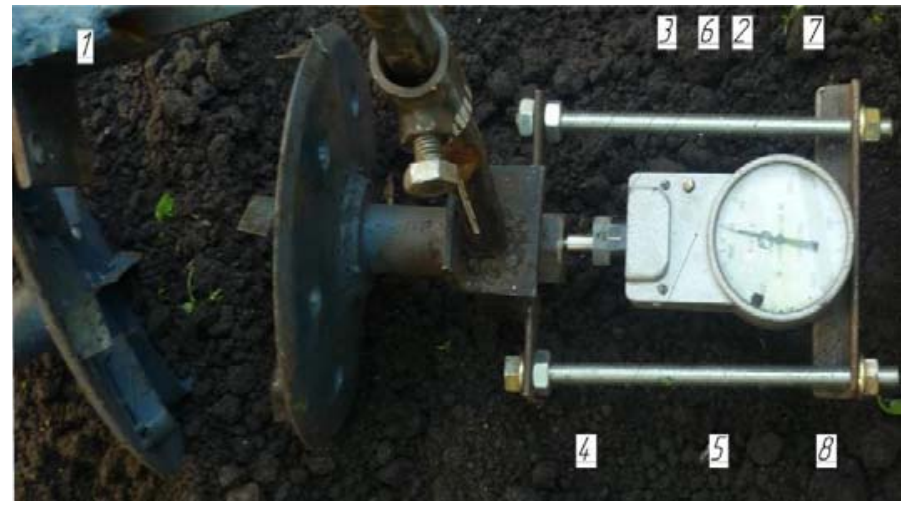

Fig. 4. Diagram of the laboratory setup for determining the rate of rotation of the disk covering element: 1 - disk covering element; 2 - adjustment screw; 3 - shaft of the disk covering element; 4 - tachymeter; 5 - mounting prop; 6 - interchangeable tip; 7 - guide; 8 -threaded joint. 


\section{Results and discussion}

Graphic representation of the results of studies to determine the rate of rotation of the disk covering element equipped with soil guides are shown on Figure 5.

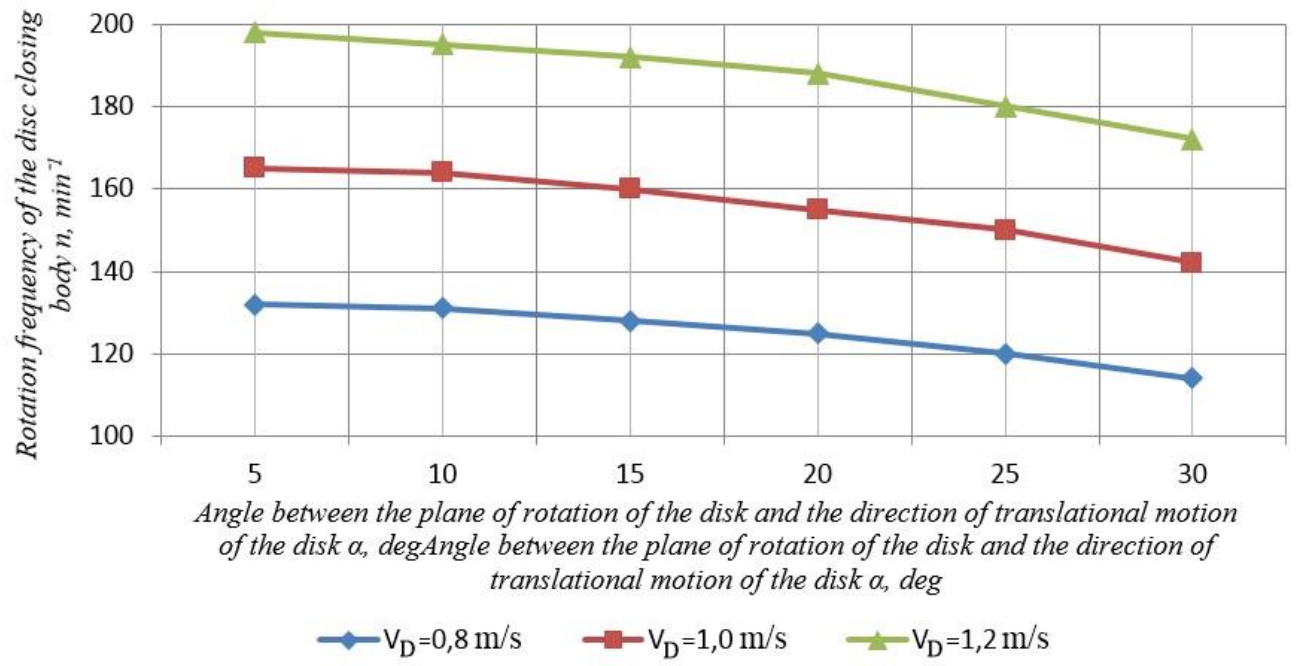

Fig. 5. Diagram of dependency of the rate of rotation of the disk covering element on the attack angle of disk $\alpha$.

Using the shown diagram, we can determine the rate of rotation of the disk covering element equipped with soil guides upon changes of attack angle of disk $\alpha$ depending on the travel speed $\mathrm{v}_{\mathrm{D}}$ of the disk.

In order to do this, after determination of the attack speed $\alpha$ of the covering element equipped with soil guides, a right line parallel to the y-axis needs to be drawn until it intersects the graph.

To determine the rate of rotation of the disk covering element, we have established an empirical dependency of the attack angle $\alpha$ of the disk on the travels speed of the covering element:

at $\mathrm{v}_{\mathrm{D}}=0.8 \mathrm{~m} / \mathrm{s}, \mathrm{n}=-5,1143 \alpha+205,4$,

at $\mathrm{v}_{\mathrm{D}}=1.0 \mathrm{~m} / \mathrm{s}, \mathrm{n}=-4,6286 \alpha+172,2$,

at $\mathrm{v}_{\mathrm{D}}=1.2 \mathrm{~m} / \mathrm{s}, \mathrm{n}=-3,6 \alpha+137,6$.

\section{Conclusions}

The results of the conducted theoretical and experimental studies of the disk covering element equipped with soil guides made it possible to determined the initial speed of flight of soil particle B whose value is mainly dependent on the structural parameters and the rate of rotation of the disk covering element. Diagram of dependence of the rate of rotation on the center-to-center distance between the covering elements was also developed.

The work was carried out with the state support of young Russian scientists - Candidates of Science MK-206.2020.8.

\section{References}

1. N.P. Laryushin, O.N. Kukharev, T.A. Kiryukhina, Science in Central Russia, 6(18), 48-58 (2015) 
2. V. Mayer, D. Vejchar, L. Pastorková, Research in Agricultural Engineering, 1, 22-31 (2017)

3. A.U. Dongre, R. Battase, S. Dudhale, V.R. Patil, D. Chavan, International Research Journal of Engineering and Technology (IRJET), 4, 1567-1570 (2017)

4. R. Farhadi, N. Sakenian, P. Azizi, Bulgarian Journal of Agricultural Science, 2, 304314 (2012)

5. J.Q. Lü, H. Sun, H. Dui, M.M. Peng, J.Y. Yu, Transactions of the CSAM, 48(11), 146155 (2017)

6. J.Q. Lü, Q.Q. Shang, Y. Yang, Z.H. Li, J.C. Li, Z.Y. Liu, Transactions of the CSAM, 47(5), 106-114 (2016)

7. A.S. Dorokhov, A.V. Sibirev, A.G. Aksenov, M.A. Mosyakov, INMATEH Agricultural Engineering, 61(2), 41-48 (2020)

8. A.V. Sibirev, A.G. Aksenov, M.A. Mosyakov, Journal of Engineering and Applied Sciences, 13(23), 10086-10091 (2018)

9. A.V. Sibirev, A.G. Aksenov, M.A. Mosyakov, Engineering technologies and systems, 29(1), 91-107 (2019)

10. A.G. Aksenov, A.V. Sibirev, AMA Agricultural Mechanization in Asia, Africa and Latin America, 51(3), 12-18 (2020)

11. A.S. Dorokhov, A.G. Aksenov, A.V. Sibirev, N.V. Sazonov, INMATEH - Agricultural Engineering, 60 (1), 107-114 (2020)

12. V.A. Ablikov, Scientific journal Cube GAU, 121 (07), 25-30 (2016)

13. O.N. Kukharev, N.P. Larushin, Research Journal of Pharmaceutical, Biological and Chemical Sciences, 9(3), 30-33 (2018)

14. S.T. Li, X.B. Chen, W. Chen, S.P, Zhu, Y.W. Li, L. Yang, Journal of Cleaner Production, 179, 55-61 (2018)

15. N. Al-Dosary Nappe Mordi, CIGR Journal, 18(2), 32-42 (2016) 